Conclusions: The in vitro results confirmed that 2ccPA had suppressing effect of cartilage degrading enzymes expression on SFs and chondrocytes, supports the hypothesis that 2ccPA might have played direct role to suppress inflammation and also protect articular cartilage in arthritic condition.

Disclosure of Interest: I. Masuda Grant/research support from: SANSHO, K. Okada: None declared, H. Yamanaka: None declared, S. Momohara: None declared

DOI: 10.1136/annrheumdis-2017-eular.5680

\section{AB0066 HUMAN MULTIPOTENTIAL STROMAL CELLS EXPRESS LOW SURFACE LEVELS OF PRO-INFLAMMATORY CYTOKINE RECEPTORS IN BONE HEALING DEFECTS}

J.J. El-Jawhari ${ }^{1}$, G. Kleftouris ${ }^{1}$, Y.M. El-sherbiny ${ }^{1}$, R. West $^{2}$, E. Jones ${ }^{1}$, P.V. Giannoudis ${ }^{1}$. ${ }^{1}$ Leeds Institute of Rheumatic and Musculoskeletal Medicine (LIRMM); ${ }^{2}$ Leeds Institute of Health Sciences, University of Leeds, Leeds, United Kingdom

Background: Osteoimmunology is an evolving field where the multipotential stromal cells (MSCs) can be considered as an important player linking immune response with bone generation. A group of pro-inflammatory cytokines including IFN- $\gamma$, TNF- $\alpha$, IL-17 and IL-1, has been proven to have a licensing effect on MSCs promoting the immunomodulatory activities of MSCs (1). Importantly, these cytokines can regulate the osteogenic differentiation capability of MSCs and in particular, IL-1 and IL-17 can enhance the MSC osteogenesis as shown in previous in vitro studies $(2,3)$. However, little is known about the role of these cytokine-MSC interactions in the bone-related diseases in humans.

Objectives: The main focus of this study was to assess if the immune-dependent licensing process of MSCs could be involved in defective bone regeneration.

Methods: We used samples of bone marrow aspirates $(n=15)$ from two groups of traumatic bone fracture patients; normal union and non-union. Bone marrow MSCs were analyzed for the surface expression of the receptors of the pro-inflammatory licensing cytokines using flowcytometry-optimized panels. Additionally, a comparison of the cytokine effect on the proliferation of cultured MSCs was compared between normal union and non-union groups using the cell proliferation XTT test.

Results: Interestingly, there were significant lower expression levels of IL-1 receptors 1 and 3 (IL-1R1 and IL-1R3) on non-union MSCs compared to normal-union MSCs $(p=0.0478$ and $p=0.0113$ respectively). Furthermore, the surface levels of TNF- $\alpha$ R1 (CD120a) were significantly lesser on non-union MSCs $(\mathrm{p}=0.0119)$. There was a clear trend of reduced expression of $\mathrm{IL}-17$ receptors (CD217) on the surface of non-union MSCs, but it was not statistically significant compared to normal-union $(p=0.0726)$. The XTT data showed a significant less proliferation index for IL-1-treated non-union MSCs compared to normal-union MSCs $(p=0.0446)$. Also, a consistent trend of lower proliferation index of non-union MSCs was detected when these cells were treated by IFN- $\gamma$, TNF- $\alpha$ or IL-17.
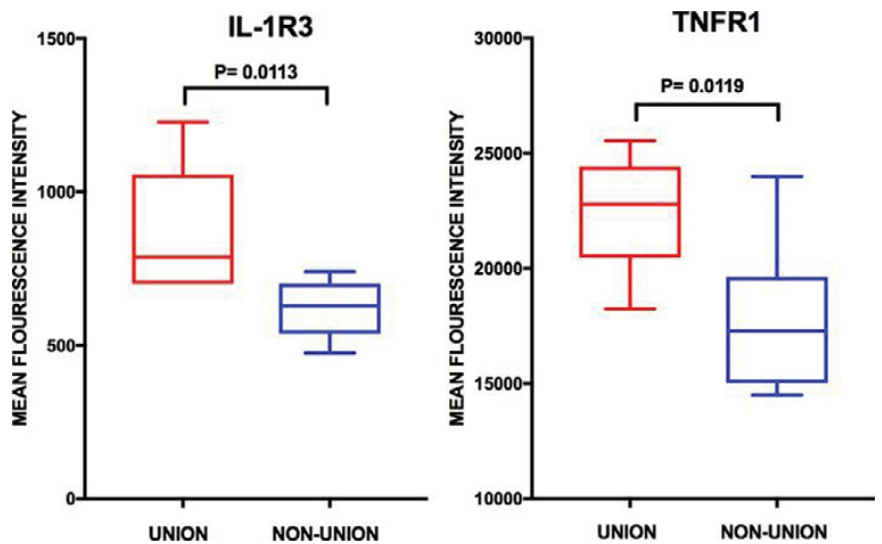

Conclusions: Together, the lower levels of the pro-inflammatory cytokine receptors indicated a possible mechanism for a defective response of non-union MSCs to the inflammatory signals (particularly IL-1). Further understating of the impact of immune-MSC interactions on human bone healing and regeneration will help to develop new therapies for musculoskeletal diseases involving osteolytic lesions. References:

[1] Mesenchymal stromal cell 'licensing': a multistep process. Krampera M. Leukemia. 2011 Sep;25(9):1408-14.

[2] Interleukin-1 $\beta$ induces differentiation of human mesenchymal stem cells into osteoblasts via the Wnt-5a/receptor tyrosine kinase-like orphan receptor 2 pathways. Sonomoto K, Yamaoka K, Oshita K, Fukuyo S, Zhang X, Nakano K, Okada Y, Tanaka Y. Arthritis Rheum. 2012 Oct:64(10):3355-63.

[3] Proinflammatory T cells and IL-17 stimulate osteoblast differentiation. Croes M, Öner FC, van Neerven D, Sabir E, Kruyt MC, Blokhuis TJ, Dhert WJ, Alblas J. Bone. 2016 Mar; 84:262-70.

Acknowledgements: This project (no. S-16-132E) was supported by the AO Foundation.
Disclosure of Interest: None declared

DOI: 10.1136/annrheumdis-2017-eular.6309

\section{AB0067 INFLUENCE OF ADIPOKINES ON DIFFERENTIATION OF SPONGIOSA-DERIVED MESENCHYMAL STROMAL CELLS FROM OSTEOPOROTIC AND OSTEOARTHRITIS PATIENTS}

L. Tsiklauri ${ }^{1}$, E. Neumann ${ }^{1}$, J. Werner ${ }^{2}$, K. Frommer ${ }^{1}$, R. Engel ${ }^{1}$, S. Rehart ${ }^{3}$, $\mathrm{S}_{\text {. Wenisch }}{ }^{2}$, U. Müller-Ladner ${ }^{1} .{ }^{1}$ Department of Internal Medicine and Rheumatology, Justus-Liebig-University Giessen, Kerckhoff-Klinik, Bad Nauheim; ${ }^{2}$ Institute of Veterinary-Anatomy, -Histology and-Embryology, Clinic of Small Animals, Justus-Liebig-University, Giessen; ${ }^{3}$ Department of Orthopedics and Trauma Surgery, Agaplesion Markus Hospital, Frankfurt, Germany

Background: Osteoporosis (OP) and osteoarthritis (OA) are two common age-related disorders leading to chronic pain and disability in elderly people. Agerelated bone loss and articular cartilage damage are associated with increased bone marrow adiposity due to a possible shift of osteogenic differentiation towards adipogenic differentiation of bone marrow mesenchymal stem cells (MSC). The differentiation of MSC into adipocytes or osteoblasts is an important determinant of bone structural integrity. Adipose tissue is an metabolically active tissue. Therefore adipocyte-derived factors -adipokines- might influence differentiation of bone marrow-derived MSC.

Objectives: The role of fat-bone interactions in the pathogenesis of OP is poorly understood. Therefore, we analyzed the presence of distinct adipokines (visfatin, resistin and leptin) in the bone marrow cavity and their effects on MSC differentiation.

Methods: Spongiosa from femoral heads was collected (hip replacement surgery of OA patients or after osteoporotic femoral neck fracture). MSC were cultured in adipogenic and osteogenic media with/without adipokines. For the transfer and differentiation of MSC on cancellous bone, bone fragments were purified and sterilized. mRNA expression of adipokines, bone marker genes, TIMPs and MMPs of stimulated MSC and bone samples were evaluated by realtime PCR. Matrix mineralization was assayed using Alizarin red $\mathrm{S}$ staining. Proinflammatory factors were measured by ELISA.

Results: Visfatin and leptin levels were increased in OP bone vs. non-osteoporotic bone $(n=14)$. In contrast to leptin and resistin, visfatin induced the secretion of proinflammatory factors (IL-6, IL-8, MCP-1) during both, osteogenic and adipogenic differentiation. Visfatin significantly increased the matrix mineralization and downregulated collagen type 1-expression (e.g. d21:-4.6-fold) in osteogenic differentiated cells. Visfatin also reduced the expression of MMP2, MMP13, RunX2, TIMP1 and TIMP2 (e.g. d21: -2.4-fold/-3.18-fold/-5.85-fold/-3.2-fold/-4.3 fold respectively) during osteogenic differentiation, but not leptin and resistin. In contrast to osteogenesis, visfatin significantly induced MMP13 expression (e.g. d21: 104-fold) during adipogenic differentiation under standard cell culture conditions. However, visfatin-induced MMP13-expression was markedly reduced during differentiation on purified autologous cancellous bone.

Conclusions: Visfatin and leptin levels were elevated in osteoporotic bone tissue. Therefore, the visfatin-mediated increase of matrix mineralization and reduction of collagen type 1 expression might lead to enhanced bone fragility and contribute to the pathogenesis of OP. Visfatin induced release of proinflammatory cytokines and dysregulated expression of MMPs and TIMPs during osteogenic and adipogenic MSC-differentiation might influence bone turnover specifically at the adipose tissue/bone interface.

Disclosure of Interest: None declared

DOI: 10.1136/annrheumdis-2017-eular.2845

\section{AB0068 HYPOXIA AND RHEUMATOID PHENOTYPE DECREASE THE CAPACITY OF SYNOVIAL FIBROBLASTS TO SUPPRESS T HELPER CELL PROLIFERATION THROUGH IDO1-MEDIATED TRYPTOPHAN CATABOLISM}

L.-O. Tykocinski ${ }^{1}$, N.-C. Kaul ${ }^{1}$, I. Adam ${ }^{2}$, S.R. Mohapatra ${ }^{2}$, S. Krienke ${ }^{1}$, C.A. Opitz ${ }^{2}$, H.-M. Lorenz ${ }^{1}$. ${ }^{1}$ Department of Medicine V, Division of Rheumatology, University of Heidelberg; ${ }^{2}$ Brain Cancer Metabolism, German Cancer Research Center (DKFZ), Heidelberg, Germany

Background: The pathogenesis of rheumatoid arthritis (RA) is linked to functional changes in synovial fibroblasts (SF) and local infiltration of T lymphocytes Increased synovial inflammation is also associated with a hypoxic joint microenvironment. Oxygen levels in the joints of RA patients are significantly decreased compared to those of osteoarthritis (OA) patients with values of about $22.5 \mathrm{mmHg}$ corresponding to ambient oxygen tensions of $3.2 \%$. So far, little is known about the effects of hypoxia on the interaction between fibroblasts and $\mathrm{T}$ lymphocytes and its implications on the pathophysiology of RA.

Objectives: The aim of this study was to compare the influence of SF from RA versus OA patients on $\mathrm{T}$ helper (Th) cell responses both under normoxic and hypoxic conditions.

Methods: SF were isolated from synovectomy tissues of OA or RA patients, Th cells were isolated from peripheral blood of RA patients or healthy donors. Cell cultures were performed under normoxic or hypoxic $\left(3 \% \mathrm{O}_{2}\right)$ conditions. Th cell proliferation was determined by $\mathrm{PKH} 26$ labelling and flow cytometry. Cytokine secretion was quantified by ELISA. Indoleamine 2,3-dioxygenase 1 